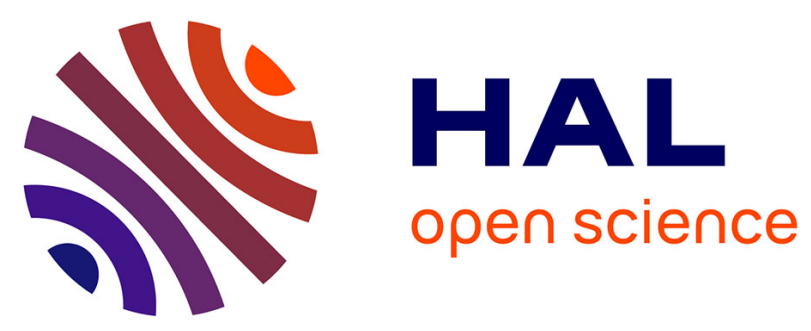

\title{
Stored energy accompanying cyclic deformation of filled rubber
}

Mohammed Taoufik Loukil, Guillaume Corvec, Eric Robin, Mathieu Miroir, Jean-Benoit Le Cam, Pierre Garnier

\section{- To cite this version:}

Mohammed Taoufik Loukil, Guillaume Corvec, Eric Robin, Mathieu Miroir, Jean-Benoit Le Cam, et al. Stored energy accompanying cyclic deformation of filled rubber. European Polymer Journal, 2018, 98, pp.448-455. 10.1016/j.eurpolymj.2017.11.035 . hal-01681197

\section{HAL Id: hal-01681197}

https://hal-univ-rennes1.archives-ouvertes.fr/hal-01681197

Submitted on 7 May 2020

HAL is a multi-disciplinary open access archive for the deposit and dissemination of scientific research documents, whether they are published or not. The documents may come from teaching and research institutions in France or abroad, or from public or private research centers.
L'archive ouverte pluridisciplinaire HAL, est destinée au dépôt et à la diffusion de documents scientifiques de niveau recherche, publiés ou non, émanant des établissements d'enseignement et de recherche français ou étrangers, des laboratoires publics ou privés. 


\title{
Stored energy accompanying cyclic
}

\section{deformation of filled rubber}

\author{
M.T. Loukil ${ }^{a, b}$ G. Corvec ${ }^{b, c}$ E. Robin ${ }^{b, c}$ M. Miroir
}

J.-B. Le Cam ${ }^{\mathrm{b}, \mathrm{c}, 1}$ P. Garnier ${ }^{\mathrm{a}}$

${ }^{a}$ PCM Technologies S.A.S., Rue René Moineau, 49123 Champtocé-sur-Loire -

France

${ }^{\mathrm{b}}$ Université de Rennes 1, Institut de Physique UMR 6251 CNRS/Université de

Rennes 1, Campus de Beaulieu, Bât. 10B, 35042 Rennes Cedex, France.

${ }^{\mathrm{c}}$ LC-DRIME, Joint Research Laboratory, Cooper Standard - Institut de Physique

UMR 6251, Campus de Beaulieu, Bât. 10B, 35042 Rennes Cedex, France.

\begin{abstract}
The hysteresis observed in the mechanical response of filled rubbers is classically assumed to be due to viscosity. In this study, a complete energy balance is carried out during cyclic deformation of a filled acrylonitrile-butadiene rubber. Results show that for the studied material, viscosity is not the preponderant contribution to the hysteresis loop: the mechanical energy brought to the material is not entirely dissipated into heat but a contrario is mainly used by the material to change its microstructure. Moreover, no significant hysteresis loop is observed in the unfilled material. Hence, the filler network stores elastic energy during its deformation, leading to a change in the internal energy. The higher the stretch applied, the higher the relative stored energy, but the higher the stretch rate applied, the lower the relative stored energy in the filler network. This has been evidenced by defining a
\end{abstract}


ratio $\gamma_{s e}$ in terms of energy. As hysteresis loop in rubbers does not systematically mean that intrinsic dissipation is produced, predicting changes in temperature, and consequently the self-heating, is not possible from the mechanical response only. To conclude, this study presents the first estimation of stored energy in a filled rubber.

Key words: Elastomer, stored energy, filler effects, IR thermography, viscosity

\section{Introduction}

Elastomers are widely used in many industries such as automotive, nuclear, or civil engineering for their high deformability, high damping and resistance to fatigue. Such properties are partly obtained by adding fillers in the rubber matrix, which increases anelastic effects in the mechanical response corresponding to the formed hysteresis loop. This hysteresis loop corresponds therefore to the dissipated part of the mechanical energy brought to rubber. Classically, this energy is assumed to be dissipated into heat due to viscosity induced by fillers. Nevertheless, several observations question this assumption:

- some non-viscous elastomers exhibit large hysteresis loop which is not due to viscosity, typically unfilled natural rubber [20]; a hysteresis loop can therefore be observed without any self-heating,

- mechanical hysteresis of certain elastomers is not time-dependent $[6,7,17$, $23]$

- if all the energy contained in the hysteresis loop were induced by viscosity, the self-heating would be much higher than that observed experimentally.

$\overline{1}$ Corresponding author jean-benoit.lecam@univ-rennes1.fr Fax : (+33) 223236111 
One can therefore wonder about the nature and the time dependency of the phenomena involved in the formation of the hysteresis loop when adding fillers to the compound. While only the viscosity is generally invoked, structural change mechanisms that store elastic energy and consequently change the internal energy of the material may also occur. In natural rubber for instance, energy storage due to strain-induced crystallization (SIC) and release during crystallite melting have different kinetics, which forms the hysteresis loop [11]. Hence, an important issue is to define if adding fillers to a rubber matrix induces similar effects. In other words, is there energy storage when the filler network deforms? If it does, is this energy released during unloading and is there a difference in kinetics between energy storage and release? These are new questions in mechanics of elastomers, which could change our vision of time dependency of the rubber behavior.

In recent studies due to Le Cam and co-workers [12], heat sources accompanying deformation of rubber have been calculated by using IR thermography and the heat diffusion equation. Characterizing heat sources enabled the authors to discuss on the calorific effects of the main phenomena involved in the deformation of rubber: Mullins effect [21], thermoelastic inversion [19], and SIC [20]. Moreover, the heat produced and absorbed was compared over each mechanical cycle in order to determine the mean intrinsic dissipation. Comparing the variation in the mean intrinsic dissipation from one cycle to another one enabled the authors to more precisely discuss on the calorific effect of the stress softening. The present study aims at characterizing the energy stored over each mechanical cycle. The approach adopted in the previous studies is therefore not sufficient, since the mechanical energy rate brought to the material has to be compared with to the energy dissipated into heat (the mean 
intrinsic dissipation), typically as recently done in case of PA6.6 by Benaarbia et al. [2, 3]. Such approach provides information of paramount importance to link microstructure to macroscopic properties and to design parts, for instance for fatigue applications.

It should be noted that the material considered in the present work is a noncrystallizable acrylonitrile-butadiene (NBR) elastomer, which avoids any coupling with energy storage effects induced by SIC.

Section 2 presents the thermodynamics framework to carry out energy balance. Section 3 presents the experimental setup. Section 4 gives the results obtained for uni-axial cyclic tensile tests in terms of mechanical, thermal and calorific responses. A complete energy balance is carried out in order to distinguish the contribution of stored and dissipated energies to the hysteresis loop. These energies are respectively associated with microstructure changes and self-heating. Concluding remarks close the paper.

\section{Thermodynamics framework}

In this section, the quantities needed for energy balances are successively introduced.

\subsection{Total strain energy density and hysteresis loop}

The total strain energy density $W_{\text {strain }}$ is the energy brought mechanically to deform the material. It corresponds to the area under the load (unload) 
strain-stress curve and is calculated as follows under uniaxial loading:

$$
W_{\text {strain }}^{\text {load }}=\int_{\text {load }} \pi d \lambda \text { and } W_{\text {strain }}^{\text {unload }}=\int_{\text {unload }} \pi d \lambda
$$

the stretch $\lambda$ is the component of the deformation gradient tensor $\mathbf{F}$ in the loading direction. It is defined as the ratio between current and initial lengths. $\pi$ is the component of the nominal stress tensor in the loading direction, defined as the force per unit of initial (undeformed) surface. If the behavior of a material is purely elastic and if the test is carried out under adiabatic loading conditions, the mechanical response obtained during a load-unload cycle is such that no hysteresis loop forms $\left(W_{\text {strain }}^{\text {load }}=W_{\text {strain }}^{\text {unload }}\right)$. If a hysteresis loop forms, the mechanical energy dissipated $W_{\text {hyst }}^{\text {cycle }}$ over one cycle is determined as follows:

$$
W_{\text {hyst }}^{\text {cycle }}=W_{\text {strain }}^{\text {load }}-W_{\text {strain }}^{\text {unload }}
$$

From this energy, a quantity $P_{\text {hyst }}^{\text {cycle }}$ is calculated in $W / \mathrm{m}^{3}$. It is obtained by dividing $W_{\text {hyst }}^{\text {cycle }}$ by the cycle duration and is therefore an energy density per time unit or an energy rate.

The last quantity calculated from the mechanical response is the strain power density at any time during the deformation. It is denoted $P_{\text {strain }}(t)$ and is defined as:

$$
P_{\text {strain }}(t)=\pi(t) \dot{\lambda}(t)
$$

\subsection{Heat source calculation in case of homogeneous tests}

Most of mechanical tests are conducted under non-adiabatic conditions. The measured temperature is therefore affected by heat diffusion, possible temperature gradients at the surface of the undeformed specimen and external heat 
sources (for instance radiations). Therefore, changes in temperature are not only due to the material deformation. In this study, a "more intrinsic" quantity, namely the heat source, is determined from the heat diffusion equation applied to the temperature field measurements. For this purpose, the thermomechanical framework described in [15] is applied. The local state axiom [4] is assumed. Any thermodynamical system out of equilibrium is considered as the sum of several homogeneous subsystems at equilibrium. The thermodynamic process, i.e. the deformation, is considered as a quasi-static phenomenon. The equilibrium state of each volume material element is defined by $n$ state variables: the absolute temperature $T$, the deformation gradient tensor $\mathbf{F}$ and $m$ $(=n-2)$ internal tensorial variables $\boldsymbol{\xi}_{\boldsymbol{\alpha}}$. Using the two principles of thermodynamics, the local form of the heat diffusion equation is written as follows [1]:

$$
\rho C \dot{T}-\operatorname{Div}\left(\boldsymbol{\kappa}_{\mathbf{0}} \operatorname{Grad} T\right)=\underbrace{\mathcal{D}_{i n t}+T \frac{\partial \boldsymbol{\Pi}}{\partial T}: \dot{\mathbf{F}}+T \sum_{\beta=1}^{m} \frac{\partial \boldsymbol{A}_{\boldsymbol{\beta}}}{\partial T}: \dot{\boldsymbol{\xi}_{\boldsymbol{\beta}}}}_{s}+R
$$

where $\boldsymbol{\kappa}_{\mathbf{0}}$ is a positive semi-definite tensor characterizing the thermal conductivity of the material. $\rho$ and $C$ are the density and the specific heat, respectively. $s$ denotes the overall heat source induced by the deformation process. The term $\mathcal{D}_{\text {int }}$ corresponds to the intrinsic dissipation (also named mechanical dissipation). The term $T \frac{\partial \Pi}{\partial T}: \dot{\mathbf{F}}$ corresponds to the heat source due to entropic and non-entropic couplings between temperature and strain, where $\boldsymbol{\Pi}$ is the nominal stress tensor. The term $T \frac{\partial \boldsymbol{A}_{\boldsymbol{\beta}}}{\partial T}: \dot{\boldsymbol{\xi}}_{\boldsymbol{\beta}}$ corresponds to the other thermomechanical couplings (for instance related to change in the material microstructure). The term $R$ is related to the external heat sources.

As temperature field provided by infrared camera is $2 \mathrm{D}$, the heat diffusion equation can be simplified by averaging the $3 \mathrm{D}$ heat diffusion equation over 
the direction corresponding to the material thickness. In the case of uniform heat sources fields, a 0D formulation of the heat diffusion equation has been proposed in [5]. Considering temperature variation $\theta$ instead of temperature, it writes:

$$
\rho C\left(\dot{\theta}+\frac{\theta}{\tau}\right)=s
$$

where $\tau$ is a time constant characterizing the heat exchanges between the specimen and its surroundings.

Remark \#1 on experimental evaluation of $\tau$ : The heat exchanges with the specimen's outside depend on the material itself (through its thermal diffusivity), its environment (ambient air and material used for the grips of the testing machine) and its geometry (through the surface-area-to-volume ratio). A value of $\tau$ has therefore to be measured for each testing configuration (machine used, environment, stretch level, etc). Experimentally, $\tau$ is usually identified for different stretch levels from a natural return of the specimen to room temperature after a heating.

\subsection{Energy balance}

The strain energy can be split into several contributions, namely the elastic, the stored and the dissipated energies. The dissipated energy is induced by irreversible processes such as viscosity and damage, the stored energy is induced by microstructure changes that modify the internal energy. Determining their relative contribution to the strain energy and therefore to the hysteresis loop provides information of paramount importance to link microstructure to macroscopic properties and to design parts, especially for fatigue applications. 
To do so, both the mechanical and the calorific responses are used. The mechanical response provides the energy $W_{\text {hyst }}^{\text {cycle }}$ (and its rate $P_{\text {hyst }}^{\text {cycle }}$ ) corresponding to the hysteresis loop. Integrating the heat source with respect to time over one cycle gives the mean intrinsic dissipation $\tilde{\mathcal{D}}_{\text {int }}$ :

$$
\tilde{\mathcal{D}}_{\text {int }}=\frac{1}{t_{\text {cycle }}} \int_{\text {cycle }} s d t
$$

The difference between $P_{\text {hyst }}^{\text {cycle }}$ and $\tilde{\mathcal{D}}_{\text {int }}$ gives the energy stored at each cycle:

$$
P_{\text {stored }}^{\text {cycle }}=P_{\text {hyst }}^{\text {cycle }}-\tilde{\mathcal{D}}_{\text {int }}
$$

Moreover, even though rubber elasticity is mainly entropic, non-entropic effects (change in the internal energy) can take place $[8,22]$. They can be investigated by comparing the strain power density with the heat source at any time during the mechanical cycles. This enables us to highlight some kinetics differences in internal energy change between loading and unloading if any.

\section{$3 \quad$ Experimental set-up}

\subsection{Materials and specimen geometry}

The considered material is an acrylonitrile butadiene rubber (NBR) with $45 \%$ ACN content. Its formulation is given in the following table. It is filled with 35 phr of N347 carbon black fillers.

Figure 1 presents the specimen geometry. It is $7.25 \mathrm{~mm}$ in wide, $1.9 \mathrm{~mm}$ in thickness and $20 \mathrm{~mm}$ in length. The cylindrical ends prevent the specimen from any slippage in the testing machine grips. Samples were elaborated according to 2 steps. The first one is a molding of a pure shear specimen by compression. 
Table 1

Chemical composition in parts per hundred rubber (phr).

\begin{tabular}{cc} 
Ingredient & Quantity \\
\hline NBR & 100 \\
Sulphur & 1.5
\end{tabular}

Zinc oxide $\mathrm{ZnO} \quad 3$

Antioxidant 2

Stearic acid $\quad 1$

Plasticizer $\quad 10$

Accelerator $/ 2$

The second step consists of cutting with a razor-blade the pure shear specimen in thin-width pieces.

\subsection{Loading conditions}

The mechanical tests were carried out using a 5543 Instron testing machine at room temperature, in the range of $23-25^{\circ} \mathrm{C}$. An overview of the experimental setup is given in Figure 2. The mechanical tests consist in applying 6 sets of 5 cycles at an increasing maximum stretch $\lambda_{\max }$ from 1.5 to 4 with a step equal to 0.5 (see Figure 3 ). The 5 cycles at a given $\lambda_{\max }$ ensure to reach the stabilized mechanical behavior. The signal shape is triangular to apply a constant loading rate. Three different loading rates were tested: $\pm 50, \pm 100$ and $\pm 200 \mathrm{~mm} . \mathrm{min}^{-1}$, which correspond to nominal strain rates equal to 0.042 
$s^{-1}, 0.083 s^{-1}$ and $0.166 s^{-1}$, respectively. They enable us to test the time dependency of the material behavior in this range of strain rates. Each test was repeated 3 times with different specimens.

\subsection{Temperature measurements}

Temperature measurements were carried out by using infrared thermography. Further information on infrared thermography in case of large deformations is provided in [10]. The infrared camera was a FLIR infrared camera (see Figure 2) equipped with a focal plane array of $640 \times 512$ pixels and detectors operating in wavelengths between 1.5 and $5.1 \mu \mathrm{m}$. The integration time was equal to $1000 \mu s$ and the acquisition frequency was set to 15 frames per second (fps). The thermal resolution or noise equivalent temperature difference is equal to $20 \mathrm{mK}$ for a temperature range between $5^{\circ} \mathrm{C}$ and $40^{\circ} \mathrm{C}$. The spatial resolution of the camera is characterized by a pixel size equal to $0.204 \mathrm{~mm}$. The surface emissivity of carbon black filled rubber is close to a black body one and is fixed at 0.94. Similar values are used in the literature for such measurement technique. The infrared camera is switched on at least 3 hours before testing in order to ensure its internal temperature to be stabilized. The calibration of camera detectors was performed with a black body using a Non-Uniformity Correction (NUC) procedure.

In the present study, the mean temperature of two zones of interest is considered. They are located at the centres of the tested and reference specimens. A suitable movement compensation technique is used to track the centre of the tested specimen (see [16] and [19] for further information on this technique). The reference specimen provides the ambient temperature changes during the 
test. In this way, the temperature variation of the tested specimen can be corrected by means of the following equation:

$$
\theta=\theta_{s p}-\theta_{\text {ref }}=\left(\left(T_{s p}(t)-T_{s p}\left(t_{0}\right)\right)-\left(\left(T_{r e f}(t)-T_{r e f}\left(t_{0}\right)\right)\right.\right.
$$

where the indices ${ }_{s p}$ and ${ }_{r e f}$ respectively stand for the tested and reference specimens.

\section{Results and discussion}

\subsection{Mechanical response}

Figure 4 presents the mechanical response of both the unfilled and the filled NBR in terms of the nominal stress in relation to the stretch for the three loading rates applied. The stress-stretch curves obtained for the unfilled NBR exhibit the classical mechanical response observed in non-crystallizable unfilled rubbers: non-linear elasticity, high stretch levels undergone, small mechanical hysteresis. When the compound is filled with carbon black, additional phenomena take place: stress-hardening at high stretch, cyclic stress-softening, namely the Mullins effect [14], an increase in the residual stretch and the mechanical hysteresis. This is classically observed in carbon black filled rubbers.

The stress-stretch response obtained for the unfilled NBR is not affected by the loading rate, which was expected. However, the material behavior remains not time-dependent when fillers are added. As mechanical hysteresis is usually assumed to be due to viscosity induced by fillers, identifying phenomena involved in this mechanical energy dissipation (the hysteresis loop) is therefore an important issue to better understand the deformation processes and the 
effects of fillers. For this purpose, a thermomechanical analysis based on quantitative calorimetry has been carried out. Such thermomechanical analysis has already been successfully performed on natural rubber [19, 20]. In this study, the authors showed that a hysteresis forms without any intrinsic dissipation, which explains why the stress-stretch response of NR is not really affected by changes in the applied loading rate. Moreover, [13] and [9] have shown that in polyurethane materials, compact or foamed, a significant part of the mechanical hysteresis is not converted into heat and is used by the material to change its microstructure.

\subsection{Thermal response}

Figure 5 depicts the temperature variation $\theta$ and the mean temperature variation $\theta_{\text {moy }}$ as a function of time for the three loading rates for the filled NBR (Figs. 5 (a) to (c)). First, results show that increasing the stretch increases the temperature variation. This means that the strain-temperature relationship is mainly governed by the entropic coupling. As expected, when the stretch level increases, the temperature variation amplitude increases. When the loading rate increases, the mean temperature variation slightly increases as well. Indeed, the mean temperature variation remains low and ranges between 0 for the lowest loading rate and $0.8^{\circ} \mathrm{C}$ for the highest one. Therefore, a part of the mechanical energy brought to deform the material is converted into heat due to viscosity and induces a self-heating. The higher the stretch rate, the higher the self-heating. Furthermore, the maximum temperature variation increases from $0.95^{\circ} \mathrm{C}$ at $50 \mathrm{~mm} \cdot \mathrm{min}^{-1}$ to $1.66^{\circ} \mathrm{C}$ at $200 \mathrm{~mm} . \mathrm{min}^{-1}$, i.e. an increase ratio of $74.74 \%$. In contrast, the nominal stress increases from $1.62 \mathrm{MPa}$ at 
$50 \mathrm{~mm} . \mathrm{min}^{-1}$ to $1.66 \mathrm{MPa}$ at $200 \mathrm{~mm} . \mathrm{min}^{-1}$, i.e. an increase ratio of $1.3 \%$. Consequently, the stretch rate has only an effect on the thermal activity as the mechanical response does not change. However, the self-heating is very small and does not strongly depend on the loading rate, which is consistent with the previous results obtained in terms of the stretch-strain relationship. Furthermore, temperature variations are not stabilized in the successive sets of 5 cycles. This is due to two main phenomena. This first one is the heat diffusion: the test is not adiabatic and the temperature variations at the beginning of the unstabilized cycles are different than at the end. It should be noted that under adiabatic conditions, the temperature variation would theoretically continuously increase without any stabilization as intrinsic dissipation is produced. The second one is due to stress softening which decreases the temperature variation amplitude, especially between the first and second cycles. In the following, heat sources are calculated to remove heat diffusion effects from the thermomechanical analysis. Thus, the stabilization of the mechanical response induces the heat source one.

\subsection{Calorific response}

Figure 6 presents the heat source $s\left(\right.$ in $\left.W \cdot m^{-3}\right)$ in relation to the stretch at \pm 50 (Fig. 6(a)), \pm 100 (Fig. 6(b)) and \pm 200 (Fig. 6(c)) mm.min ${ }^{-1}$. The red color is used for the loading phase and the blue one for the unloading phase. Heat sources are positive during the loading phases and negative during the unloading phases. This is due to preponderant entropic effects previously discussed. Heat sources have quasi-linear evolution with the stretch. This is consistent with the calorific response of filled non-crystallizable rubbers such as carbon 
black filled SBR [18]. No change in slope is detected in the heat source curve as observed in crystallizing elastomers [20]. Nevertheless, a slight shift towards positive heat sources is observed, which signs the effects of the intrinsic dissipation produced at each cycle. In this case, the total heat source is the sum of the heat sources due to elastic couplings and intrinsic dissipation at any time of the cycle. This will be quantified and more precisely discussed in the following. A strong decrease in the heat source is observed between the first and the next loadings for each set of maximum stretch. This is due to stress softening effects [14]. As heat sources are superimposed during the unloadings at a given maximum stretch applied, the intrinsic dissipation due to the Mullins effect could be deduced from the difference between the first two loadings in terms of heat sources. Such characterization of the Mullins effect has already been performed in [21]. As a consequence, the corresponding temperature variation amplitude decreases between the first and second loadings, as shown previously in Fig. 5. The maximum heat source increases from $0.9310^{5} \mathrm{~W} . \mathrm{m}^{-3}$ at $50 \mathrm{~mm} . \mathrm{min}^{-1}$ to $4.6410^{5}$ at $200 \mathrm{~mm}^{\mathrm{min}} \mathrm{m}^{-1}$. However, the nominal stress does not evolve with the stretch rate (from $1.62 \mathrm{MPa}$ at $50 \mathrm{~mm} \cdot \mathrm{min}^{-1}$ to $1.66 \mathrm{MPa}$ at $200 \mathrm{~mm} . \mathrm{min}^{-1}$, see Fig. 4). This means that the effects of yiscosity are very small. Nevertheless, a significant hysteresis loop is observed in the mechanical response. To further discuss on this effect, the energy rate corresponding to the mechanical hysteresis $\left(P_{\text {hyst }}^{\text {cycle }}\right)$ and the mean intrinsic dissipation $\left(\tilde{\mathcal{D}}_{\text {int }}\right)$ are plotted in the same diagram in Figure 7 for the three loading rates. As explained previously, the intrinsic dissipation is determined by integrating the heat source with respect to time over each mechanical cycle. It should be noted that when no intrinsic dissipation occurs, numerical processing of images can lead to intrinsic dissipation close to zero, but negative. In this case, the value of the intrinsic dissipation reported in 
Figure 7 is set at 0 .

Whatever the stretch rate considered, the stabilized intrinsic dissipation ${ }^{2}$ tends to decrease when the stretch is increased. For the lowest stretch rate, no intrinsic dissipation is detected from the third set of applied maximum stretch.

By comparing the values of $P_{\text {hyst }}^{\text {cycle }}$ and $\tilde{\mathcal{D}}_{\text {int }}$ at each cycle, a significant part of the mechanical hysteresis is not dissipated into heat but is used by the material to change its microstructure ${ }^{3}$. Part of the mechanical energy brought to deform the material is therefore stored elastically during deformation. Such results were already obtained for polyurethane by [9] and[13]. Moreover, the difference between the two curves increases with the stretch, meaning that the higher the stretch, the higher the energy stored.

To further discuss on the relative contribution of the energy stored in the hysteresis loop of rubbers, authors propose to define a ratio $\gamma_{s e}$, written in terms of energy as follows:

$$
\gamma_{s e}=\frac{W_{\text {stored }}^{\text {cycle }}}{W_{\text {hyst }}^{\text {cycle }}}
$$

- if $\gamma_{s e}$ tends to 0 , no energy is stored during the deformation. The whole hysteresis loop is due to the intrinsic dissipation,

- if $\gamma_{s e}$ tends to 1 , the whole hysteresis loop is due to energy stored and no intrinsic dissipation is detected. This is typically the case in unfilled natural rubber [20].

\footnotetext{
2 obtained from the second cycle of each set

3 else, the stretch rate should have an effect on the material stiffness, which is not observed in the mechanical response (see Figure 4)
} 
$\gamma_{s e}$ is plotted in relation with the stretch and for the three loading rates applied in Figure 8. First, these diagrams show that the relative energy stored increases with the stretch until to be close to the whole energy contained in the hysteresis loop at the highest stretch applied $\left(\gamma_{s e}=1\right)$. The relative energy stored decreases with the stretch rate even though the amount of energy stored increases. Such relation provides information of first importance for rubber part design, especially when considering an increase in the stretch, which usually means in the stretch rate in anti-vibratory system applications.

\subsection{How do microstructure changes contribute to the hysteresis loop?}

The previous analysis enables us to highlight and to quantify the energy stored by the material. Nevertheless, it does not provide any information on how microstructure changes contribute to the hysteresis loop. Indeed, investigating microstructure changes requires to compare heat source $s$ with strain power density $P_{\text {strain }}$ during the mechanical cycles. Three cases can be obtained:

- if $P_{\text {strain }}=s$ : no internal energy variation occurs, the material does not store energy,

- if $P_{\text {strain }}>s$ : internal energy changes occur, the material stores energy,

- if $P_{\text {strain }}<s$ : preponderant exothermal effects (latent heat) occur, whatever the material stores energy or not.

It should be noted that change in internal energy is not sufficient to form a hysteresis loop, a typical result being the elastic behavior of metals. Without intrinsic dissipation production, a difference in kinetics of internal energy changes between loading and unloading is necessary to form a hysteresis loop. 
Figures $9(\mathrm{a}), 9(\mathrm{~b})$ and $9(\mathrm{c})$ gives the heat source and strain power density for the three loading rates applied. First of all, it is observed that during loading, the difference between the two curves, which is induced by internal energy variations, increases with the stretch. This difference is not significant during unloading. This highlights a difference in internal energy changes, i.e. in energy storage and release, between loading and unloading. As the hysteresis loop in unfilled NBR is very small, this phenomenon is only due to fillers. Therefore, the filler network does not store and release energy with the same kinetics during loading and unloading.

\section{Conclusion}

In this study, mechanical and thermal measurements have been carried out during cyclic deformation of a carbon black filled nitrile rubber. The total strain energy and the intrinsic dissipation have been calculated for each cycle. Results show that viscosity is not the preponderant contribution to the hysteresis loop: the mechanical energy brought to the material is not entirely dissipated into heat but a contrario is mainly used by the material to change its microstructure. A ratio $\gamma_{s e}$ has been proposed to relativise the energy stored at each cycle. Calculating this ratio as a function of the stretch and the stretch rate has shown that the relative energy stored increases with the stretch and decreases with the stretch rate. This ratio is of a first importance for the rubber part design as it characterizes the greater or lesser ability of the rubber to absorb mechanical energy without damaging. This has numerous consequences on the mechanical behaviour, typically for fatigue. Finally, the filler network stores elastic energy during its deformation, leading to a change 
in the internal energy. This elastic energy is released with a different kinetics during unloading, which is the main origin of the hysteresis loop.

\section{Acknowledgements}

The authors thank the PCM company for providing the materials and for supporting financially this work. The authors thank the National Center for Scientific Research (MRCT-CNRS and MI-CNRS) and Rennes Metropole for supporting this work financially.

\section{References}

[1] Balandraud, X. and Le Cam, J.-B. (2014). Some specific features and consequences of the thermal response of rubber under cyclic mechanical loading. Archive of Applied Mechanics, 84, 773-788.

[2] Benaarbia, A., Chrysochoos, A., and Robert, G. (2014). Kinetics of stored and dissipated energies associated with cyclic loadings of dry polyamide 6.6 specimens. Polymer Testing, 34, 155-167.

[3] Benaarbia, A., Chrysochoos, A., and Robert, G. (2015). Influence of relative humidity and loading frequency on the PA6.6 thermomechanical cyclic behavior: Part II. Energy aspects. Polymer Testing, 41, 92-98.

[4] Boccara, N. (1968). Les principes de la thermodynamique classique. In PUF coll. SUP.

[5] Chrysochoos, A. and Louche, H. (2000). An infrared image processing to analyse the calorific effects accompanying strain localisation. Int J Eng Sci, 38, 1759-1788. 
[6] D’Ambrosio, P., De Tommasi, D., Ferri, D., and Puglisi, G. (2008). A phenomenological model for healing and hysteresis in rubber-like materials. International Journal of Engineering Science, 46, 293-305.

[7] Dorfmann, A. and Ogden, R. (2003). A pseudo-elastic model for loading, partial unloading and reloading of particle-reinforced rubber. International Journal of Solids and Structures, 40, 2699-2714.

[8] Flory, P. J. (1961). Thermodynamics relation for high elastic materials. Transactions of the Faraday Society, 57, 829-838.

[9] Lachhab, A., Robin, E., Le Cam, J.-B., Mortier, F., Tirel, Y., and Canevet, F. (2017). Thermomechanical analysis of closed-cell polymeric foams subjected to cyclic loading: anelasticity, self-heating and stress-induced crystallization. Polymer, 126, 19-28.

[10] Le Cam, J.-B. (2012). A review of the challenges and limitations of fullfield measurements applied to large heterogeneous deformations of rubbers. Strain, 48, 174-188.

[11] Le Cam, J.-B. (2017). Energy storage due to strain-induced crystallization in natural rubber: the physical origin of the mechanical hysteresis. Polymer, 127, $166-173$.

[12] Le Cam, J. B., Samaca Martinez, J. R., Balandraud, X., Toussaint, E., and Caillard, J. (2015). Thermomechanical Analysis of the Singular Behavior of Rubber: Entropic Elasticity, Reinforcement by Fillers, Strain-Induced Crystallization and the Mullins Effect. Experimental Mechanics, 55, 771782.

[13] Mott, P., Giller, C., Fragiadakis, D., Rosenberg, D., and Roland, C. (2016). Deformation of polyurea: Where does the energy go? Polymer, 105, $227-233$.

[14] Mullins, L. (1948). Effect of stretching on the properties of rubber. Rubber 
Chemistry and Technology, 21, 281-300.

[15] Nguyen, Q., Germain, P., and Suquet, P. (1983). Continuum thermodynamics. J Appl Sci, 50, 1010-1020.

[16] Pottier, T., Moutrille, M.-P., Le Cam, J.-B., Balandraud, X., and Grédiac, M. (2009). Study on the use of motion compensation technique to determine heat sources. application to large deformations on cracked rubber specimens. Experimental Mechanics, 49, 561-574.

[17] Rey, T., Chagnon, G., Favier, D., and Le Cam, J.-B. (2014). Hyperelasticity with rate-independent microsphere hysteresis model for rubberlike materials. Computational Materials Science, 90, 89-98.

[18] Samaca Martinez, J. R., Le Cam, J.-B., Balandraud, X., Toussaint, E., and Caillard, J. (2013a). Filler effects on the thermomechanical response of stretched rubbers. Polymer testing, 32, 835 - 841 .

[19] Samaca Martinez, J. R., Le Cam, J.-B., Balandraud, X., Toussaint, E., and Caillard, J. (2013b). Mechanisms of deformation in crystallizable natural rubber. part 1: Thermal characterization. Polymer, 54, $2717-2726$.

[20] Samaca Martinez, J. R., Le Cam, J.-B., Balandraud, X., Toussaint, E., and Caillard, J. (2013c). Mechanisms of deformation in crystallizable natural rubber. part 2: Quantitative calorimetric analysis. Polymer, 54, 2727 $-2736$

[21] Samaca Martinez, J. R., Le Cam, J.-B., Balandraud, X., Toussaint, E., and Caillard, J. (2014). New elements on mullins effect: a thermomechanical analysis. European Polymer Journal, 55, 98-107.

[22] Treloar, L. R. G. (1973). The elasticity and related properties of rubbers. Reports on Progress in Physics, 36, 755.

[23] Vandenbroucke, A., Laurent, H., Hocine, N. A., and Rio, G. (2010). A Hyperelasto-Visco-Hysteresis model for an elastomeric behaviour: Experi- 
mental and numerical investigations. Computational Materials Science, 48, 495-503. 


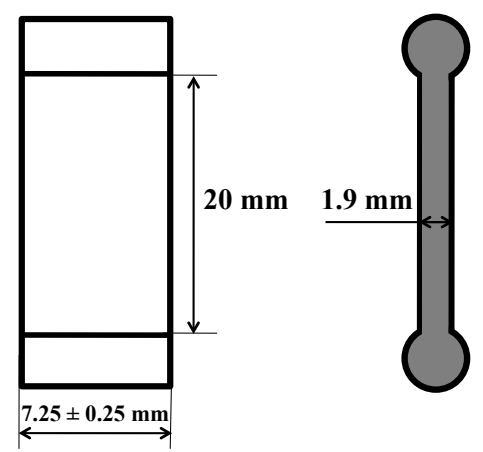

Fig. 1. Specimen geometry 


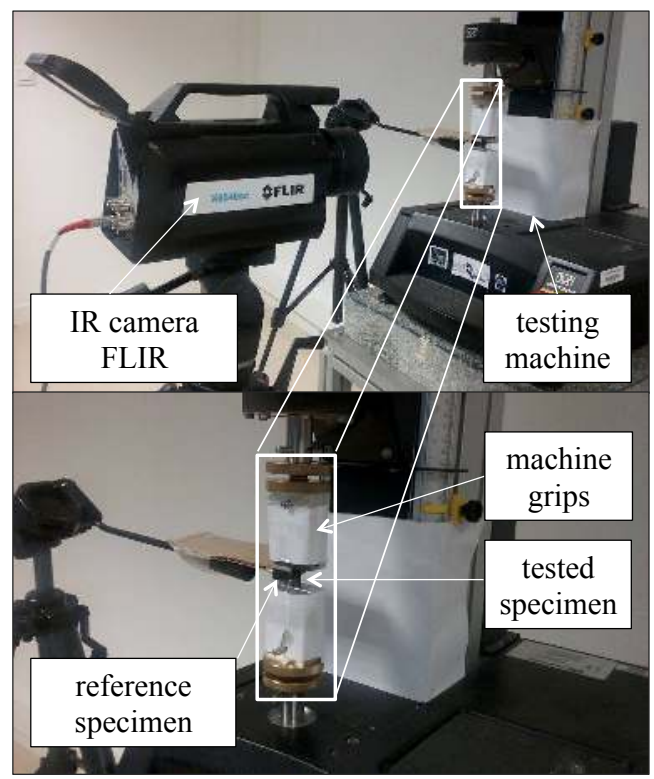

Fig. 2. Experimental setup 


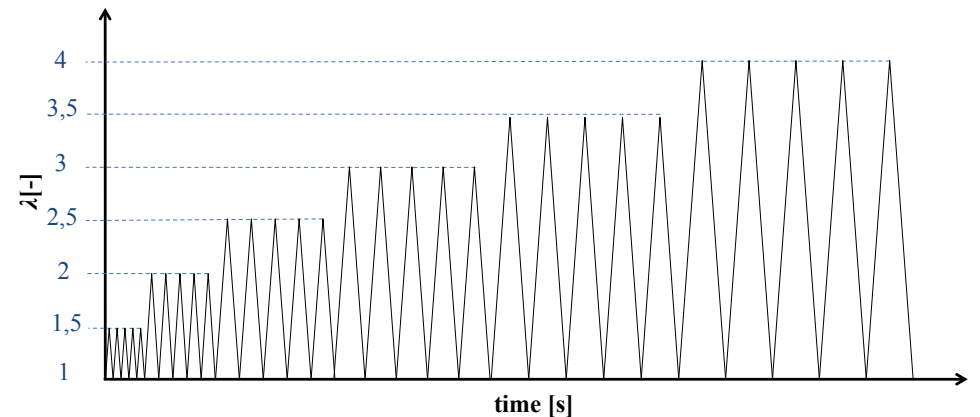

Fig. 3. Loading condition 


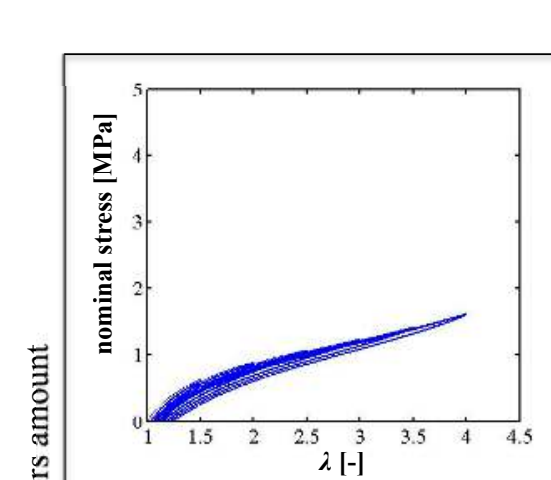

(a) unfilled NBR at $50 \mathrm{~mm} \cdot \mathrm{min}^{-1}$
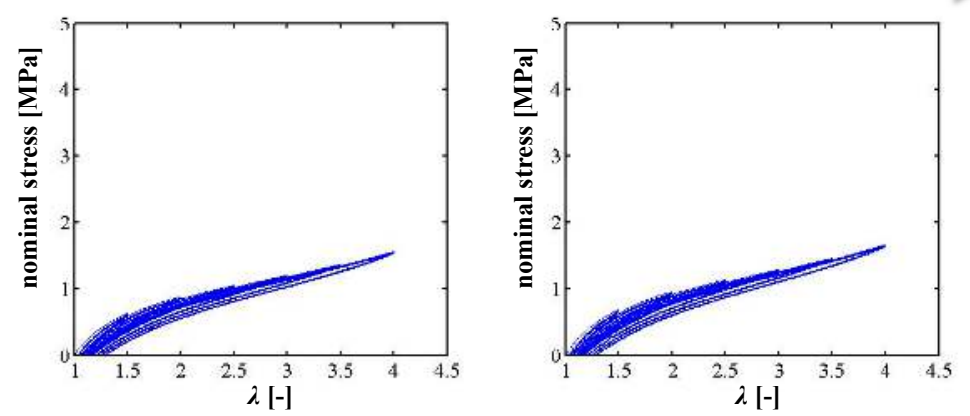

(a) unfilled NBR at 50 mm.min

(b) unfilled NBR at $100 \mathrm{~mm} \cdot \mathrm{min}^{-1}$

(c) unfilled NBR at $200 \mathrm{~mm} \cdot \mathrm{min}^{-1}$

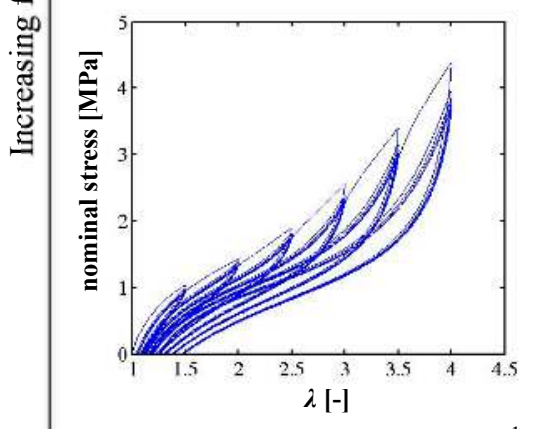

(d) filled NBR at $50 \mathrm{~mm} \cdot \mathrm{min}^{-1}$

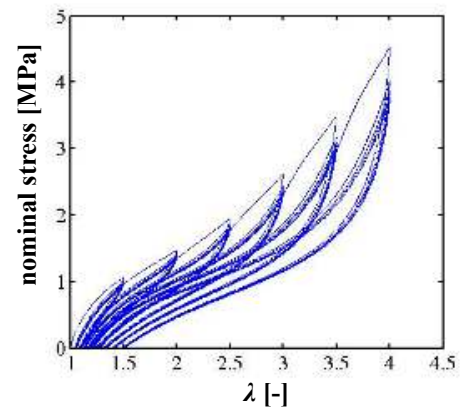

(e) filled NBR at $100 \mathrm{~mm} \cdot \mathrm{min}^{-1}$

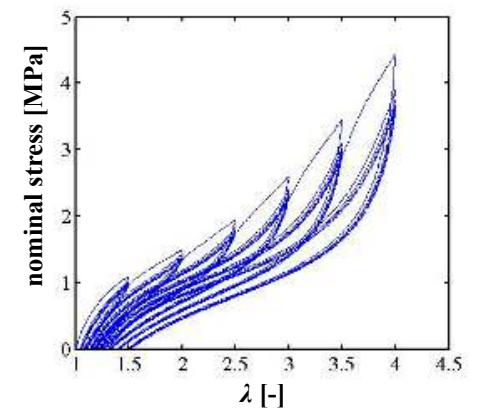

(f) filled NBR at $200 \mathrm{~mm} \cdot \mathrm{min}^{-1}$

Fig. 4. Mechanical response of NBR during cyclic uniaxial tensile tests 


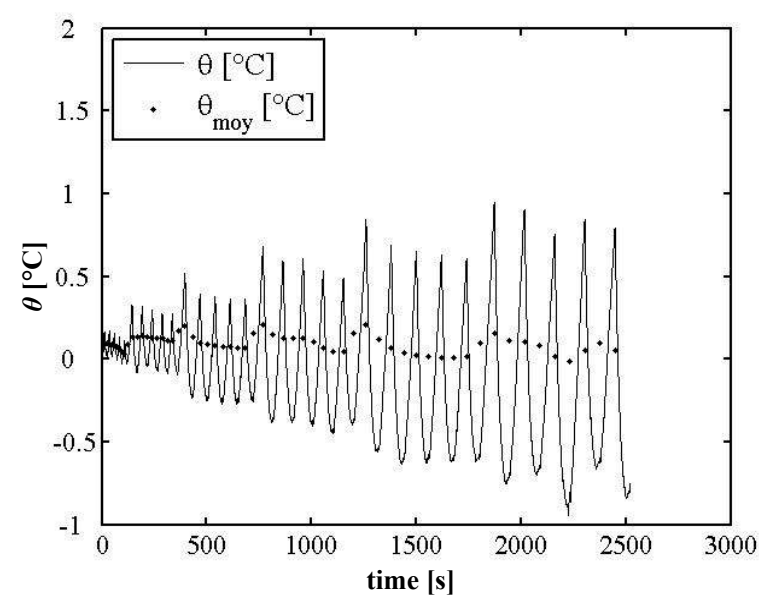

(a) $50 \mathrm{~mm} \cdot \mathrm{min}^{-1}$

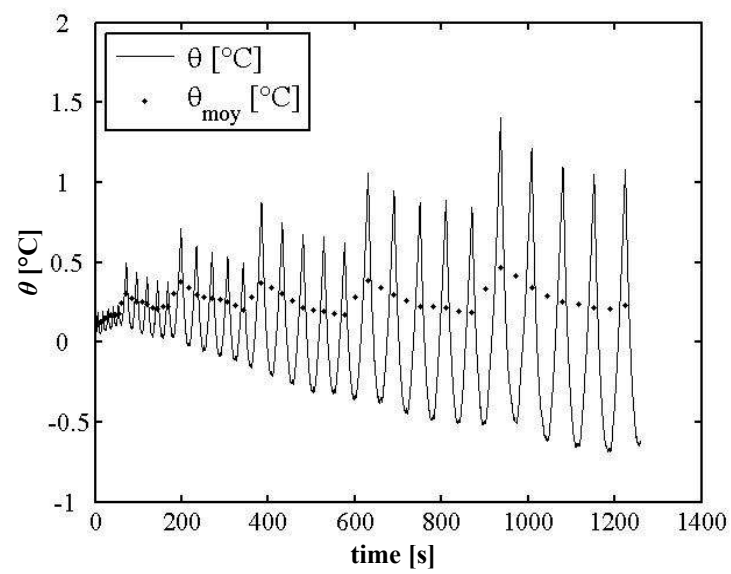

(b) $100 \mathrm{~mm} \cdot \mathrm{min}^{-1}$

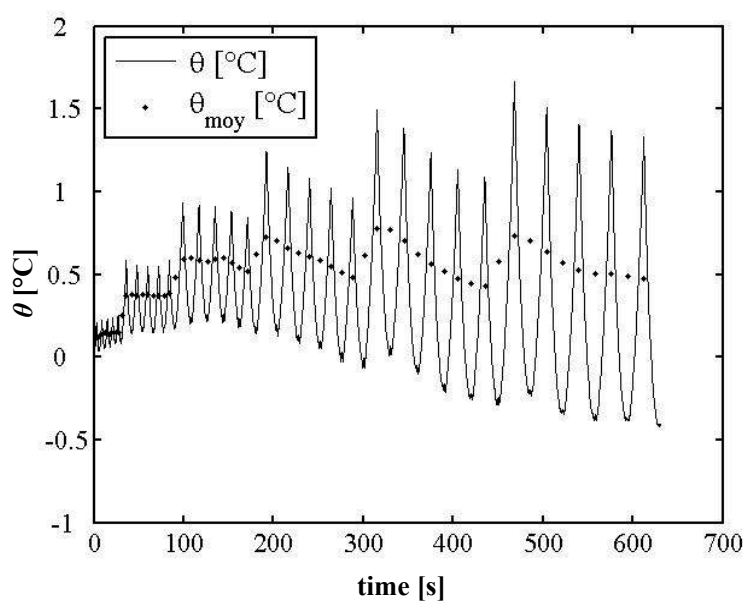

(c) $200 \mathrm{~mm} \cdot \mathrm{min}^{-1}$

Fig. 5. Temperature variations versus time in filled NBR 


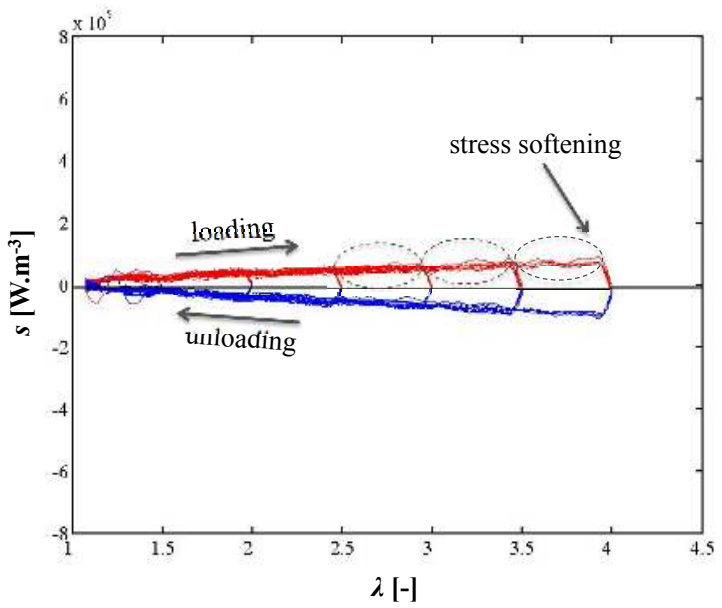

(a) $50 \mathrm{~mm} \cdot \mathrm{min}^{-1}$

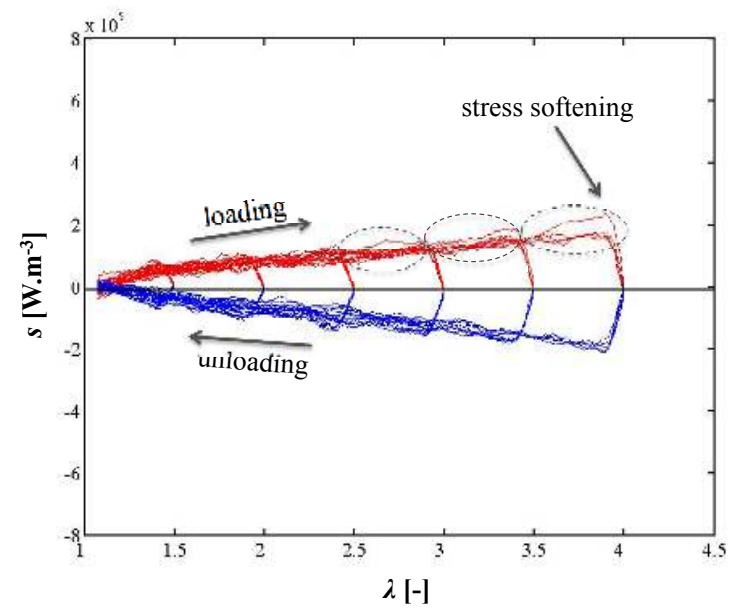

(b) $100 \mathrm{~mm} \cdot \mathrm{min}^{-1}$

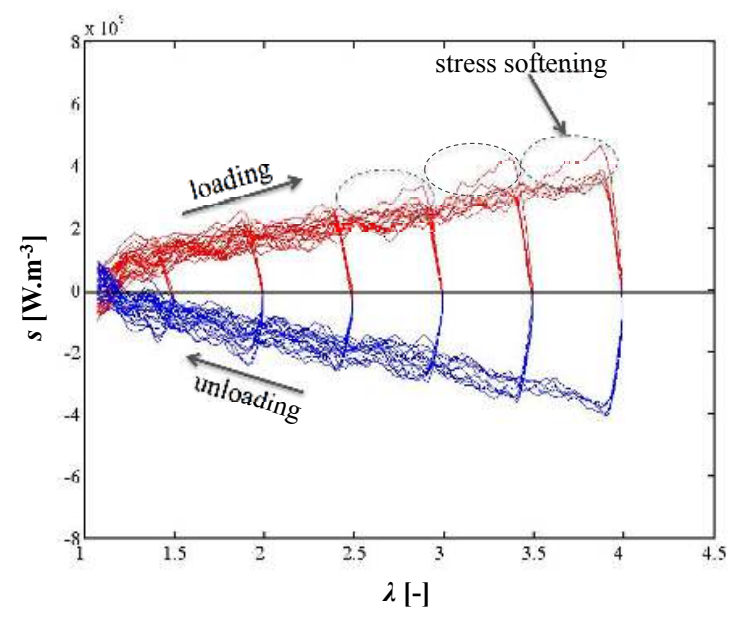

(c) $200 \mathrm{~mm} \cdot \mathrm{min}^{-1}$

Fig. 6. Heat sources versus stretch obtained for filled NBR 


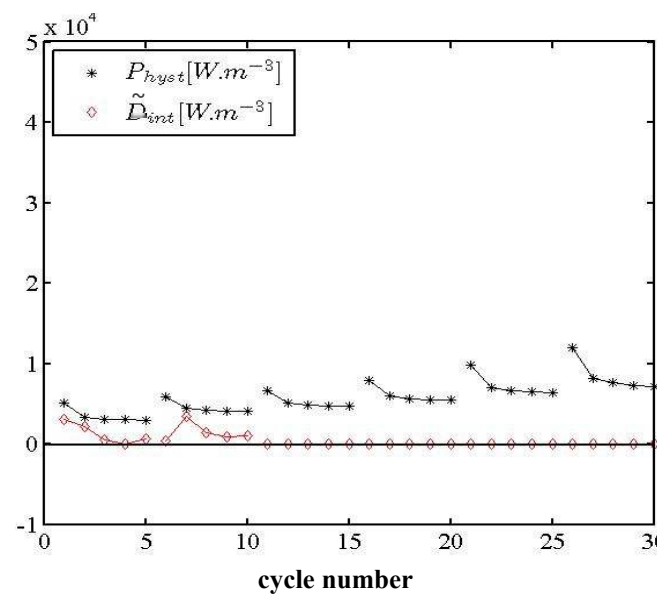

(a) $50 \mathrm{~mm} \cdot \mathrm{min}^{-1}$

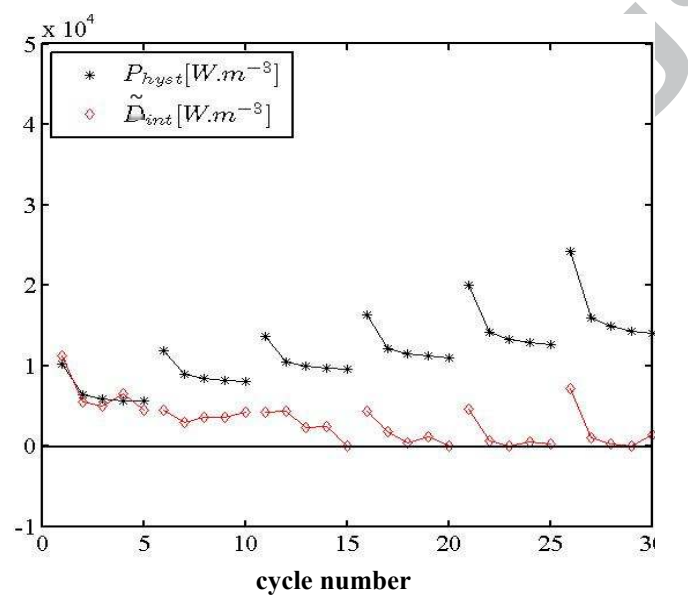

(b) $100 \mathrm{~mm} \cdot \mathrm{min}^{-1}$

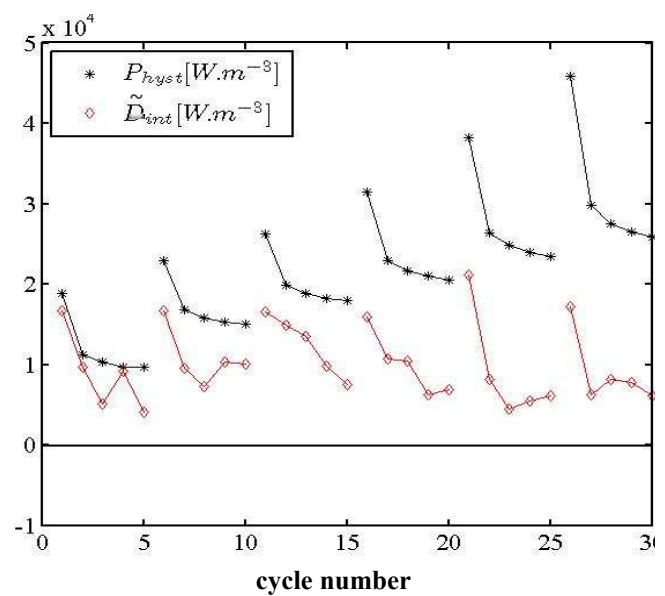

(c) $200 \mathrm{~mm} \cdot \mathrm{min}^{-1}$

Fig. 7. Cyclic evolutions of $\tilde{\mathcal{D}}_{\text {int }}$ and $P_{\text {hyst }}^{\text {cycle }}$ obtained for filled NBR during cyclic uniaxial tensile tests 

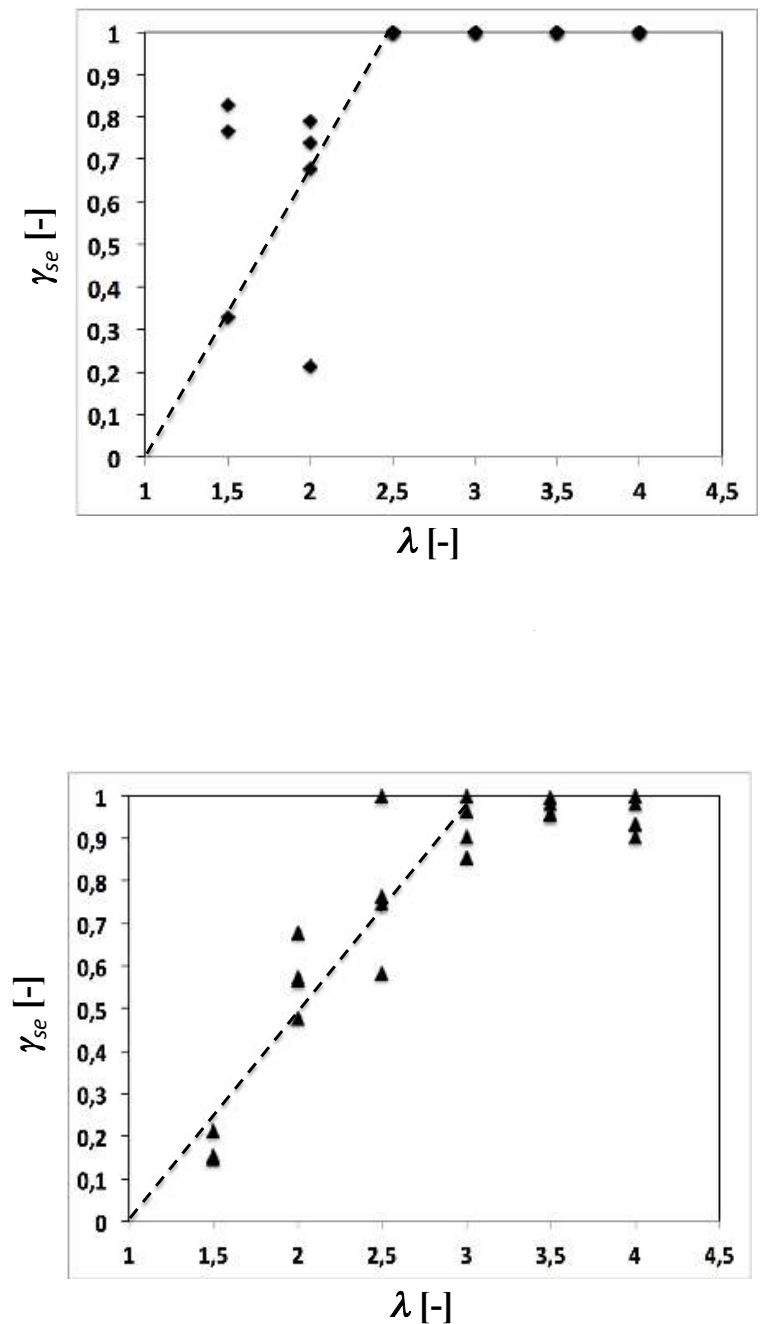

(h) $1 \mathrm{n} \cap \mathrm{mm} \min ^{-1}$

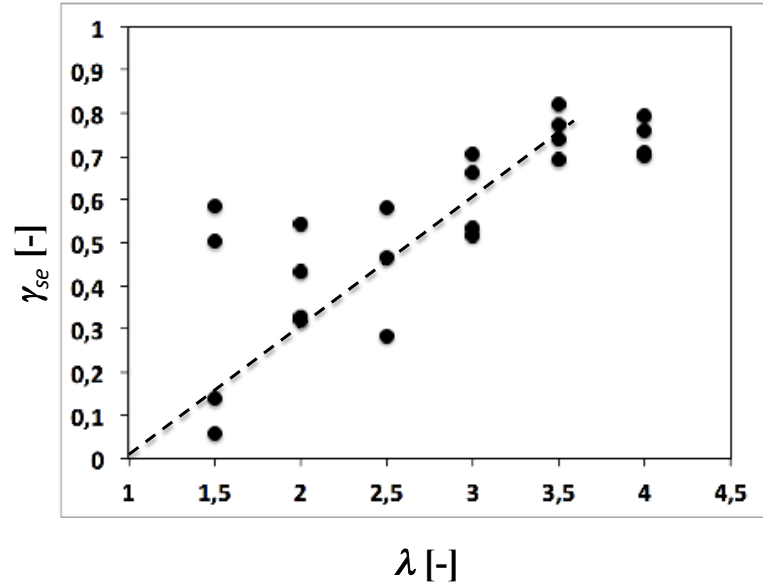

(c) $200 \mathrm{~mm} \cdot \mathrm{min}^{-1}$

Fig. 8. $\gamma_{s e}$ versus stretch for filled NBR 29 

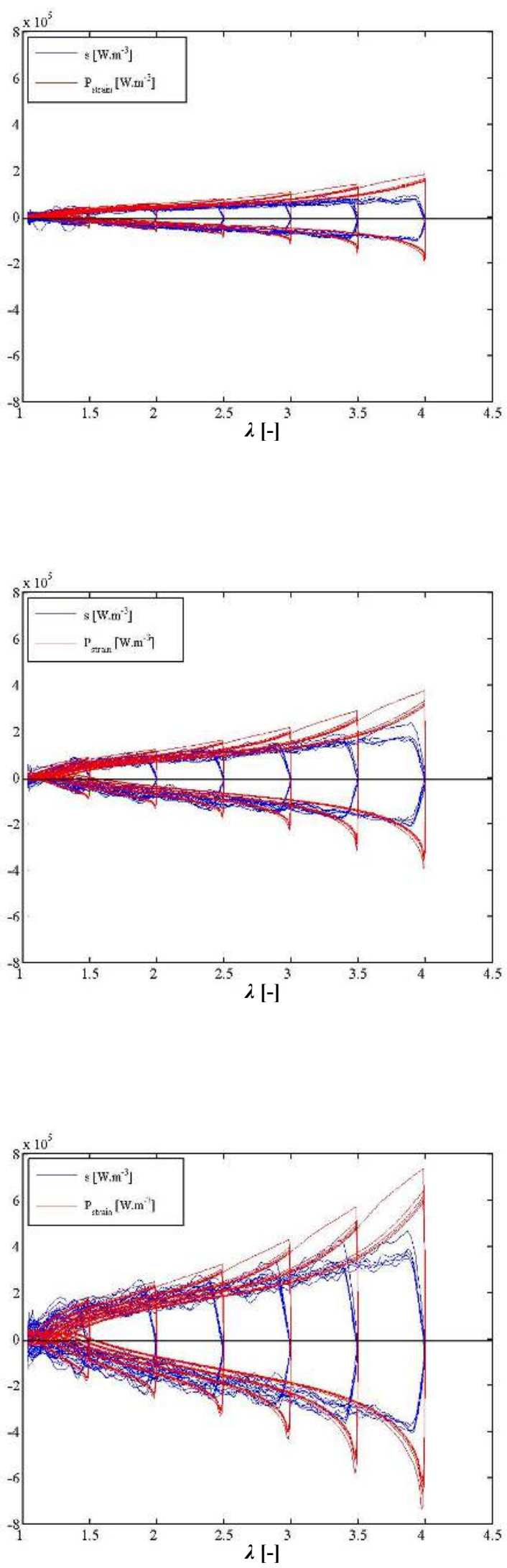

(c) $200 \mathrm{~mm} \cdot \mathrm{min}^{-1}$

Fig. 9. Heat source and strain power density versus stretch for filled NBR 
A complete energy balance is carried out during cyclic deformation of NBR.

Viscosity is not the preponderant contribution to the hysteresis loop.

Filler network stores energy during deformation and releases it during unloading.

The energy is released with a different kinetics, this is why a hysteresis loop forms.

A new ratio is proposed for quantifying energy stored in rubbers. 

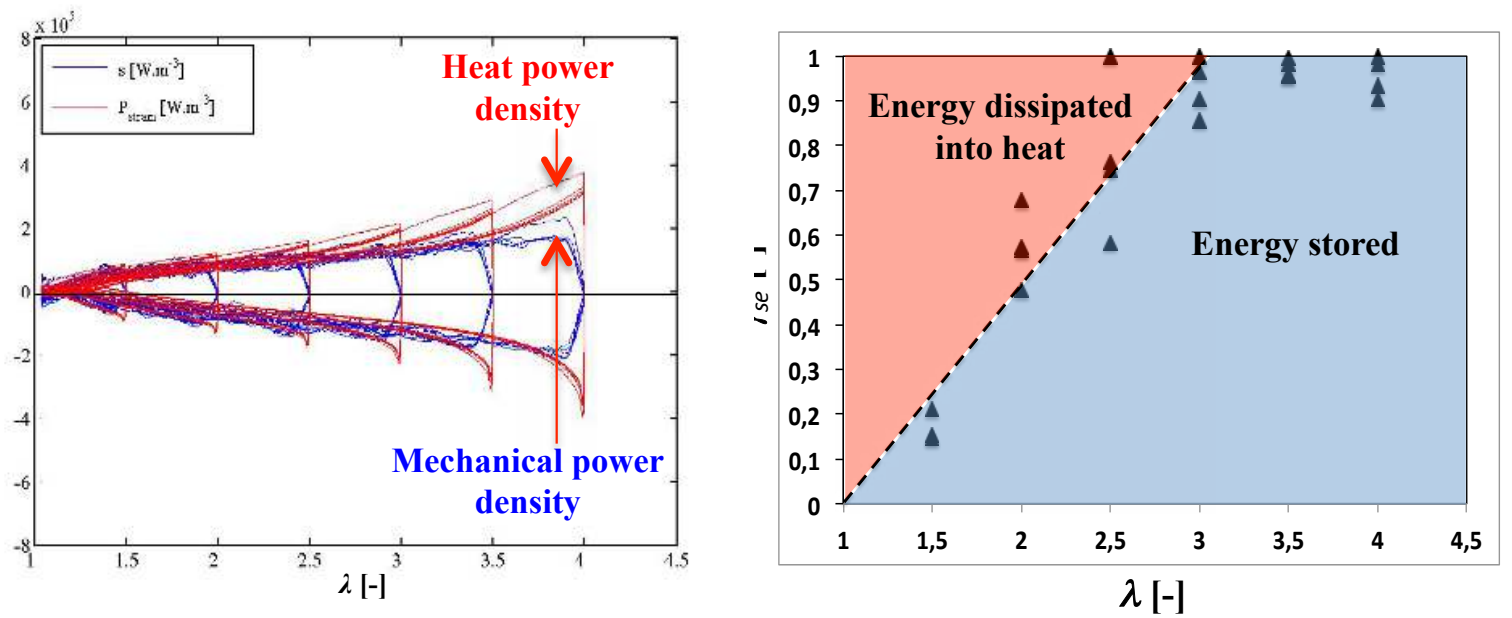